\title{
Estimation of Objective and Risk-neutral Distributions based on Moments of Integrated Volatility
}

\author{
René Garcia, Marc-André Lewis ${ }^{\dagger}$ \\ and \\ Éric Renault $\ddagger$ \\ CIRANO \\ 2020 University, 25th floor \\ Montréal,Québec \\ CANADA (H3A-2A5)
}

October 2, 2001

\section{Working Paper Preliminary and Incomplete}

\begin{abstract}
In this paper, we present an estimation procedure which uses both option prices and high-frequency spot price feeds to estimate jointly the objective and risk-neutral parameters of stochastic volatility models. This procedure is based on series expansions of option prices and implied volatilities and on a methodof-moment estimation that uses analytical expressions for the moments of the integrated volatility. This results in an easily implementable and rapid estimation technique.
\end{abstract}

${ }^{*}$ Université de Montréal, CIRANO and CRDE; E-mail: rene.garcia@umontreal.ca

†Banque Nationale du Canada and CIRANO; E-mail: lewism@cirano.umontreal.ca

${ }^{\ddagger}$ Université de Montréal, CIRANO, CRDE and Crest-Insee; E-mail: eric.renault@umontreal.ca 


\section{Introduction}

Continuous-time models and associated arbitrage-free pricing techniques are a convenient black-box approach to derivative asset pricing whereby continuoustime processes for asset prices are combined with an absence of arbitrage argument to obtain the prices of derivative assets. Therefore, statistical inference on continuous-time models of asset prices can and should combine two sources of information, namely the price history of the underlying assets on which derivative contracts are written and the price history of the derivative securities themselves. However, the joint use of primitive and derivative asset price data raises several difficulties put forward in Renault [36].

First, the maintained assumption of no arbitrage opportunities imposes a tight relationship between the primitive and the derivative asset prices. According to Harrison and Kreps [25], under this assumption, the value of any asset can be computed as the expectation of its discounted terminal payoff with respect to an equivalent martingale or so-called risk-neutral measure. When this argument is applied to contingent claim pricing, a second difficulty appears for statistical modeling. A joint model has to be specified, not only for the objective probability distribution which governs the random shocks observed in the economy, but also for the risk-neutral probability distribution which is the mathematical tool used to compute derivative asset prices as expectations of discounted payoffs. Since the two distributions have to be equivalent, the density process of the martingale measure is linked to the objective probability distribution through an integral martingale representation which includes the innovations associated with the primitive asset price processes and the risk premia associated with these sources of uncertainty. It can also include state variables, observable or latent, which affect the drift and diffusion coefficients of the primitive assets and the corresponding risk premia.

If there are no state variables, the drift and diffusion coefficients are deterministic functions of time and of the current value of the underlying asset spot price. In this case, no arbitrage pricing will lead to express any derivative asset price, say a European option price, as a deterministic function of the underlying asset spot price. Therefore, an integrated time-series analysis of spot and option prices is bound to fail since observed asset prices are never conformable to such deterministic relationships. More precisely, if $K$ time series of option prices are observed jointly with the spot price, any multivariate time series analysis of spot and option prices would require the introduction of at least $K$ state variables.

The main contribution of this paper is to propose a new methodology for such an integrated analysis. It is based on simple generalized method-of moment (GMM) estimators of both the parameters of the asset price and state variable 
processes and the corresponding risk premia. To focus on the issue of the joint specification of an objective probability distribution and a risk-neutral one, we will restrict ourselves to the case of one state variable which will capture the stochastic feature of the volatility process of the underlying asset. We will adopt the affine diffusion model popularized by Duffie, Pan and Singleton [16] where volatility is parameterized as follows:

$$
\begin{aligned}
\sigma_{t}^{2} & =\alpha+\xi V_{t} \\
d V_{t} & =k\left(\theta-V_{t}\right) d t+\gamma \sigma_{t} d W_{t}^{\sigma}
\end{aligned}
$$

where $V_{t}$ is a latent state variable with an innovation governed by a Brownian motion $W_{t}^{\sigma}$.. This innovation can be correlated (with a coefficient $\rho$ ) with the innovation of the primitive asset price process governed by $W_{t}^{S}$ :

$$
\frac{d S_{t}}{S_{t}}=\mu_{t} d t+\sigma_{t} d W_{t}^{S}
$$

In a seminal paper, Hull and White [27] have shown that, in the particular case where $\rho=0$, the arbitrage-free option price is nothing but a conditional expectation of the Black and Scholes [8] price, where the constant volatility parameter $\sigma^{2}$ is replaced by the so-called integrated volatility process: $\frac{1}{T-t} \mathcal{V}_{t, T}=\frac{1}{T-t} \int_{t}^{T} \sigma^{2}(s) d s$ and where the conditional expectation is computed with respect to the risk-neutral conditional probability distribution of $\mathcal{V}_{t, T}$ given $\sigma_{t}$. Our method of moments will be based on analytical expressions for the moments of this integrated volatility to estimate the parameter vector of the vector $\beta=(\kappa, \theta, \gamma, \alpha, \xi, \rho)$. These expressions were derived recently by Lewis [31] using a recursive method. Heston [26] has extended the analytical treatment of this option pricing formula to the case where $\rho$ is different from zero, allowing for leverage effects and the presence of risk premia.

However, with or without correlation, the option pricing formula involves the computation of a conditional expectation of a highly nonlinear integral function of the volatility process. To simplify this computation, we propose to use an expansion of the option pricing formula in the neighborhood of $\gamma=0$, as in Lewis[30], which corresponds to the Black-Scholes deterministic volatility case. The coefficients of this expansion are well-defined functions of the conditional moments of the joint distribution of the underlying asset returns and integrated volatilities, which we also derive analytically. These analytical expansions will allow us to compute very quickly implied volatilities which are functions of the parameters of the processes and of the risk premia. A two-step GMM approach using intraday returns for computing approximate integrated volatilities (the objective part of the estimation) and option prices for computing implied volatilities 
(the risk-neutral part of the estimation) allow us to recover the volatility risk premia $\lambda$. The main attractive feature of our method is its simplicity once analytical expressions for the various conditional moments of interest are available. The great advantage of the affine diffusion model is precisely to allow an analytical treatment of the conditional moments of interest.

Duffie, Pan and Singleton [16] have extended these computations to the case of affine jump-diffusion models (where jumps are captured by Poisson components), while Barndorff-Nielsen and Shephard [6] have put forward the so-called Ornstein-Uhlenbeck-like processes with a general Levy innovation. The general statistical methodology that we develop in this paper could be extended to these more general settings if a specification is chosen for the risk premia of the various jump components. Bollerslev and Zhou (2001) have developed such a GMM approach based on the first two moments of integrated volatility to estimate the objective parameters of stochastic volatility and jump-diffusion models.

Only recently has the joint estimation of risk-neutral and objective parameters been considered in the literature. Renault, Pastorello and Touzi $[34,35]$ proposed an iterative estimation procedure that used option and returns information to provide an estimate of the objective parameters in the absence of risk premia. The method proposed here can be seen as an extension of this work. More recently, in a series of two papers, Chernov, Ghysels, Gallant and Tauchen $[11,12]$ proposed a two-step procedure involving seminonparametric densities and a calibration by simulation, using the Efficient Method of Moments (EMM). Finally, Pan [33] proposed a joint estimation using option and spot prices for jump-diffusion models. Her approach is probably the closest to ours; lots of attention is devoted to the identification of risk premia and the involved moment conditions are derived analytically. However, unlike our method, the option and spot data sets in her procedure have the same measure frequency, and therefore it doesn't use the available high-frequency feeds. Another advantage of our method is its simplicity and computational efficiency; Chernov's et al. and Pan's methods are much more complex to implement and the estimation procedure they propose is computationally more intensive so that estimation is much slower.

The rest of the paper is organized as follows. In Section 2, we will present our method, and show how it can be separated in blocks to which we will devote the subsequent sections of this paper. Section 3 will be devoted to the estimation of $\hat{\beta}$ from the high-frequency return measures, using analytic expressions for the moments of the integrated volatility. In Section 4, we will show how one can use option prices expansions to define model-specific implied volatilities as power series in the volatility of volatility parameter $\gamma$, and how these implied volatilities can lead to the estimation of $\tilde{\beta}(\hat{\beta}, \lambda)$. In the following section, the two 
estimates will be compared in order to obtain the volatility risk premium estimate $\hat{\lambda}$. Finally, in Section 6, we will consider a numerical example: the Heston model, for we which we simulated price-volatility trajectories and associated option prices. The whole estimation procedure is performed over these data sets and the results of these estimations are presented and discussed. Finally, in Appendix, computational details are given.

\section{An outline of the method}

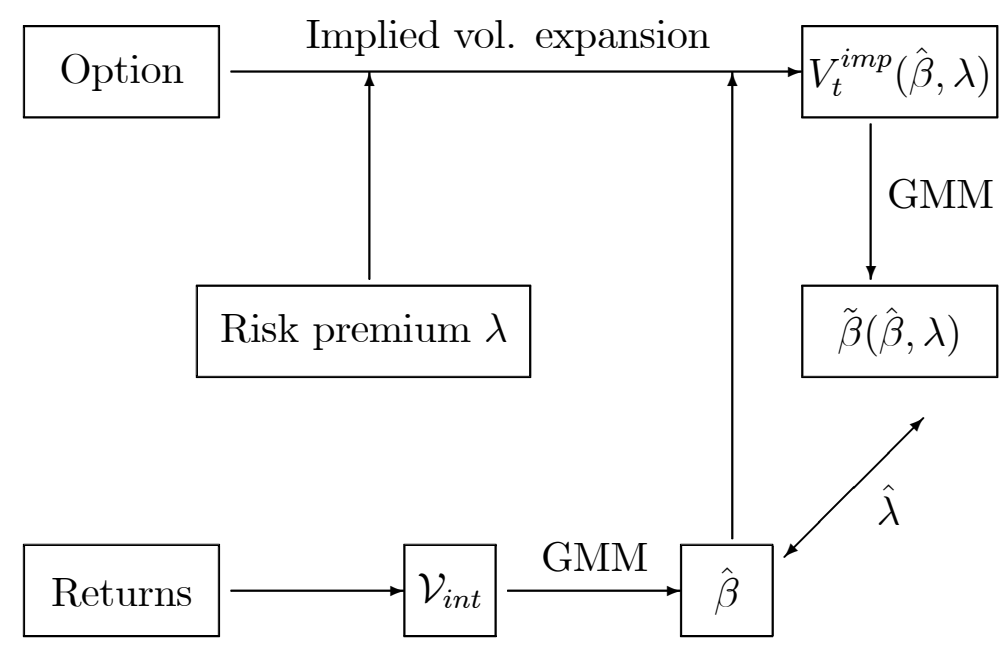

Figure 1: This flowchart presents an overview of our estimation procedure. Given option prices and high-frequency measures of the returns of the underlying asset, and for a given value of the risk premium $\lambda$, one is able to obtain objective parameters estimations $\wedge \beta$ and $\sim \beta(\beta, \lambda)$.

As stated in the introduction, two different but equivalent sets of bivariate stochastic processes are to be considered here. The objective process is taken to be the affine stochastic volatility process

$$
d\left[\begin{array}{c}
S_{t} \\
V_{t}
\end{array}\right]=\left[\begin{array}{c}
\mu_{t} S_{t} \\
\kappa\left(\theta-V_{t}\right)
\end{array}\right] d t+\sqrt{\alpha+\xi V_{t}}\left[\begin{array}{cc}
S_{t} & 0 \\
\gamma \rho & \gamma \sqrt{1-\rho^{2}}
\end{array}\right]\left[\begin{array}{l}
d W_{t}^{1} \\
d W_{t}^{2}
\end{array}\right]
$$

where $S_{t}$ and $V_{t}$ are the price and volatility processes. The affine qualification comes from the form of the volatility appearing in the returns process, a form 
which was first studied by Duffie and Kan [15]. The risk neutral process is taken to be

$$
d\left[\begin{array}{l}
S_{t} \\
V_{t}
\end{array}\right]=\left[\begin{array}{c}
r_{t} S_{t} \\
\kappa^{*}\left(\theta^{*}-V_{t}\right)
\end{array}\right] d t+\sqrt{\alpha+\xi V_{t}}\left[\begin{array}{cc}
S_{t} & 0 \\
\gamma \rho & \gamma \sqrt{1-\rho^{2}}
\end{array}\right]\left[\begin{array}{c}
d \tilde{W}_{t}^{1} \\
d \tilde{W}_{t}^{2}
\end{array}\right]
$$

where we assumed that only the parameters $\kappa$ and $\theta$ were modified in the passage from one measure to the other. This is in accord with the risk premia structure proposed by Heston [26], which generalizes, in the affine case, to

$$
\begin{gathered}
\kappa^{*}=\kappa-\lambda \\
\kappa^{*}\left(\alpha+\xi \theta^{*}\right)=\kappa(\alpha+\xi \theta),
\end{gathered}
$$

the volatility risk premium being parametrized by $\lambda$. For such models, the objective parameters to be estimated are ${ }^{1}$

$$
\beta=(\kappa, \theta, \gamma, \alpha, \xi, \rho)
$$

In order to define the risk-neutral set of parameters, one must have the additional parameter $\lambda$, since we shall assume the short rate $r_{t}$ to be observed. By abuse of language, we will often refer to the risk-neutral parameters as the vector $(\beta, \lambda)$, which is simply related to the set

$$
\beta^{*}=\left(\kappa^{*}, \theta^{*}, \gamma, \alpha, \xi, \rho\right) .
$$

In the next sections, we will show that high-frequency measures of returns can be used to measure the integrated volatility $\mathcal{V}_{t, T}$. In a recent paper, Lewis [31] proposes a method to compute conditional moments of the integrated volatility in affine stochastic volatility models. Using these, it is possible to construct a set of moment conditions $f_{1}\left(\mathcal{V}_{t, T}, \beta\right)$, which is such that

$$
\mathrm{E}\left[f_{1}\left(\mathcal{V}_{t, T}, \beta\right)\right]=0 .
$$

Moreover, as we will present, it is possible to define model-specific implied volatilities $V_{t}^{i m p}\left(\beta, \lambda,\left\{c_{o b s}\right\}\right)$, with $\left\{c_{o b s}\right\}$ being the set of observed option prices. These implied volatilities, that are not to be confounded with Black-Scholes implied volatilities, are defined to be the point-in-time volatility which gives, for given values of the risk-neutral parameters $\beta^{*}$, the observed option price. As we will discuss, it can be used to construct a second set of moment conditions

\footnotetext{
${ }^{1}$ Since the drift term $\mu_{t}$ does not matter for option pricing purposes, we do not specify it explicitely. Moreover, the inference method we will use for the objective parameters is robust to its specification.
} 
$f_{2}\left(V_{t}^{i m p}\left(\beta, \lambda,\left\{c_{o b s}\right\}\right), \tilde{\beta}\right)$, which depends on the values of the risk-neutral parameters $\beta, \lambda$ taken to define the implied volatilities and on the parameters associated to the implied volatility process, which we will denote $\tilde{\beta}$. Note that if the model is correctly specified, one expects $\tilde{\beta}$ and $\beta$ to be equal. This second set of moment conditions obeys the usual requirement

$$
\mathrm{E}\left[f_{2}\left(V_{t}^{i m p}\left(\beta, \lambda,\left\{c_{o b s}\right\}\right), \tilde{\beta}\right)\right]=0,
$$

Since we want $\tilde{\beta}=\beta$, it is thus possible to construct the set of moment conditions

$$
\mathrm{E}\left[\begin{array}{c}
f_{1}\left(\mathcal{V}_{t, T}, \beta\right) \\
f_{2}\left(V_{t}^{i m p}\left(\beta, \lambda,\left\{c_{o b s}\right\}\right), \beta\right)
\end{array}\right]=0
$$

which we would like to use to estimate the objective parameters $\beta$ and the risk premium $\lambda$, both of them providing the risk-neutral parameters $\beta^{*}$. There are at least two estimation methods one could use.

Such a set of moment conditions can be estimated efficiently using a two-step GMM approach. Namely, one first restricts his attention to the first set of moment conditions, from which it is possible to obtain an estimate $\hat{\beta}$ of the objective parameters. Then, one considers the simplified set of moment conditions

$$
\mathrm{E}\left[\begin{array}{c}
f_{1}\left(\mathcal{V}_{t, T}, \beta\right) \\
f_{2}\left(V_{t}^{i m p}\left(\hat{\beta}, \lambda,\left\{c_{o b s}\right\}\right), \beta,\right)
\end{array}\right]=0
$$

The important point here is that Gouriéroux, Monfort and Renault [23] have shown that there is no efficiency loss when comparing the system of moment conditions (4) to the one in (3).

In the first version of this paper, we intend to simplify this approach by using sequential rather than two-step GMM. That is, we will use the first set of moment conditions to obtain the estimate $\hat{\beta}$.

$$
\mathrm{E}\left[f_{1}\left(\mathcal{V}_{t, T}, \beta\right)\right]=0 \longrightarrow \hat{\beta} .
$$

Given a value of $\lambda$, we will then use the estimate $\hat{\beta}$ to obtain $\tilde{\beta}(\hat{\beta}, \lambda)$;

$$
\mathrm{E}\left[f_{2}\left(V_{t}^{i m p}\left(\hat{\beta}, \lambda,\left\{c_{o b s}\right\}\right), \beta\right)\right]=0 \longrightarrow \tilde{\beta}(\hat{\beta}, \lambda) .
$$

Finally, by defining a suitable metric, a task for which the results from indirect inference can be used, we will minimize the distance between the two sets of objective parameters estimate $\hat{\beta}$ and $\tilde{\beta}(\hat{\beta}, \lambda)$ in order to estimate $\hat{\lambda}$;

$$
\hat{\lambda}=\min _{\lambda}\|\hat{\beta}-\tilde{\beta}(\hat{\beta}, \lambda)\| \text {. }
$$


From the above discussion, such an approach appears to be suboptimal. However, since the computation of the implied volatility in the $f_{2}$ set of moment conditions is based on moments of integrated volatility, it is not obvious that the efficiency gain in the two-step approach will be large. Moreover, the method proposed here is simple to implement and permits rapid estimations. It also allows for different measure frequencies for the returns and option data, something which is likely to happen when dealing with empirical data, since the underlying asset is usually more liquid than the option, so that higher-frequency quotations are available for the former. In this spirit, it is probably more suited for practical use than the two-step GMM approach, to which we nonetheless wish to devote more attention in a subsequent evolution of this paper. A schematic view of how our sequential GMM approach works can be found in Figure 1.

\section{Estimating objective parameters from the returns}

From the recent works of Andersen and Bollerslev [3] and Barndorff-Nielsen and Shephard [6], we know that high-frequency intraday data on returns can be used to obtain indirect information on the otherwise unobservable volatility process. The logarithmic price of an asset is assumed to obey the stochastic differential equation

$$
d p_{t}=\mu\left(p_{t}, V_{t}, t\right) d t+\sqrt{V_{t}} d W_{t},
$$

where $V_{t}$ is the squared-volatility process (which could be stochastic, particularly of the affine type we discussed above) and $W_{t}$ is a standard brownian motion. Note that from now on, we shall restrict our attention to the $\alpha=0, \xi=1$ case. If the drift and diffusion coefficients are sufficiently regular to guarantee the existence of a unique strong solution to the SDE, then, by the theory of quadratic variation, we have

$$
\lim _{N \rightarrow \infty} \sum_{i=1}^{N}\left[p_{t+\frac{i}{N}(T-t)}-p_{t+\frac{i-1}{N}(T-t)}\right]^{2} \longrightarrow \int_{t}^{T} V_{s} d s \equiv \mathcal{V}_{t, T}
$$

and $\mathcal{V}_{t, T}$ is referred to as the integrated volatility of the process $V_{t}$ from time $t$ to $T$. Andersen et al. $[4,5]$ offer a characterization of the distributional features of daily realized returns volatilities constructed from high-frequency five-minutes returns for foreign exchange and individual stocks. The finiteness of the number of measures induces a systematic error in the integrated volatility measure, and, in fact, the quadratic variation estimator will be a biased estimator of the integrated volatility if the drift term is not zero, this bias falling as the 
number of measures increases. Bollerslev and Zhou [9] use such an aggregation of returns to obtain integrated volatility time series from which they estimate by GMM the parameters of Heston's stochastic volatility model [26]. They base their estimation on a set of conditional moments of the integrated volatility, where they add to the basic conditional mean and second moment various lagone and lag-one squared counterparts. In constructing estimates of the objective parameters of the stochastic volatility process, we follow their basic approach but introduce a new set of moment conditions involving higher moments of the integrated volatility, in particular its skewness. Lewis [31] derives analytically all conditional moments of the integrated volatility for the class of affine stochastic volatility models (which includes the Heston [26] and Hull-White [27] models ${ }^{2}$.

Some attention has to be devoted to information sets. Following the notation of Bollerslev and Zhou, we shall define the filtration $\mathcal{F}_{t}=\sigma\left\{V_{s}, s \leq t\right\}$, that is, the sigma algebra generated by the instantaneous volatility process. Our moment conditions for the integrated volatility are originally conditional on this filtration. Since only the integrated volatility is observable, we need to introduce the discrete filtration $\mathcal{G}_{t}=\sigma\left\{\mathcal{V}_{s-1, s}, s=0,1,2, \cdots, t\right\}$, which is the sigma algebra of observed integrated volatilities. Evidently, the filtration $\mathcal{G}_{t}$ is nested in the finer $\mathcal{F}_{t}$. This enables one to rewrite moment conditions in terms of the coarser filtration using the law of iterated expectations: $\mathrm{E}\left[\mathrm{E}\left(\cdot \mid \mathcal{F}_{t}\right) \mid \mathcal{G}_{t}\right]=\mathrm{E}\left(\cdot \mid \mathcal{G}_{t}\right)$. More specifically, we use the following set of moment conditions:

$$
f_{t}(\beta)=\left[\begin{array}{c}
\mathcal{V}_{t+1, t+2}-\mathrm{E}\left[\mathcal{V}_{t+1, t+2} \mid \mathcal{G}_{t}\right] \\
\mathcal{V}_{t+1, t+2}^{2}-\mathrm{E}\left[\mathcal{V}_{t+1, t+2}^{2} \mid \mathcal{G}_{t}\right] \\
\mathcal{V}_{t+1, t+2}^{3}-\mathrm{E}\left[\mathcal{V}_{t+1, t+2}^{3} \mid \mathcal{G}_{t}\right]
\end{array}\right]
$$

The three conditional moment restrictions (7) can be expressed in terms of observed integrated volatilities because we have (see Appendix B) closed form formulas for $\mathrm{E}\left[\mathcal{V}_{t+1, t+2}^{k} \mid \mathcal{G}_{t}\right], k=1,2,3$, in terms of $\mathrm{E}\left[\mathcal{V}_{t, t+1}^{k} \mid \mathcal{G}_{t}\right], k=1,2,3$. We use each of the resulting three orthogonality conditions with two choices of instruments. That is, for the moment condition involving $\mathrm{E}\left[\mathcal{V}_{t+1, t+2}^{k} \mid \mathcal{G}_{t}\right]$, we use both a constant and $\mathcal{V}_{t-1, t}^{k}$ as instrumental variables. This thus results in six unconditional moment restrictions ${ }^{3}$.

\footnotetext{
${ }^{2}$ Duffie, Pan and Singleton [16] provide analytical expressions for the instantaneous volatility process for such models. Bollerslev and Zhou [9] derived analytical expressions for the mean and variance of the integrated volatility in Feller-type volatility models. To our knowledge, higher moments of the integrated volatility were not previously computed.

${ }^{3}$ Due to the MA(1) structure of the error terms in (7), the optimal weighting matrix for GMM estimation entails the estimation of the variance and only the first-order autocorrelations of the moment conditions.
} 
We report in Table 1 the results of simulation experiments based on values for the parameters of the Hull and White model (which is the special case of our general affine model (37) with $\alpha=\rho=0$ and $\xi=1$ ):

$$
\begin{aligned}
& d S_{t}=\mu S_{t} d t+S_{t} \sqrt{V_{t}} d W_{t}^{1} \\
& d V_{t}=\kappa\left(\theta-V_{t}\right) d t+\gamma \sqrt{V_{t}} d W_{t}^{2} .
\end{aligned}
$$

Note that compared to the general model (37), we have set the correlation factor to zero. This makes our results directly comparable to the Monte Carlo study of Bollerslev and Zhou [9] since we use the same set of parameters. As can be seen in Table 1, our set of moment conditions provides somewhat less biased estimates of the parameters, especially the mean-reversion $\kappa$ parameter, while RMSE's are of the same order except for $\gamma^{4}$. For the purpose of this paper, it suffices to observe that our methodology provides good estimates of the true parameters. Moreover, since we dispose of all conditional moments of integrated volatility for the whole class of affine stochastic volatility models, we can easily extend this method-of-moment approach to more complicated processes for the returns and volatilities, whether the two processes are correlated or not. This is a simplified approach compared to the various estimation strategies proposed in the literature ${ }^{5}$

We will denote by $\hat{\beta}$ the estimate of the parameter vector $\beta=(\kappa, \theta, \gamma)^{\prime}$. Let us now see how option data can be used to link the objective and risk-neutral densities.

\section{Using Implied volatilities to link objective and risk-neutral parameters}

Even though it usually leads to incorrect option pricing, the Black-Scholes formula is still a central tool in the derivative industry, mainly through the widespread use of the Black-Scholes implied volatility whose moneyness dependency

\footnotetext{
${ }^{4}$ Note that our set of moment conditions is much more limited than the one used by Bollerslev and Zhou (2001), where additional moment conditions capture the dynamics of the integrated volatility process.

${ }^{5}$ These include the Markov Chain Monte Carlo methods proposed by Jacquier et al.[28], Eraker [19], Kim et al.[29] and Elerian et al[17]; the Simulated Method of Moments (SMM) of Duffie and Singleton[14]; the indirect inference method of Gouriéroux, Monfort and Renault[22]; the Efficient Method of Moments (EMM) of Gallant and Tauchen[21] and Gallant and Long[20]; the non-parametric series expansion of Aït-Sahalia[1] and Stanton[38]; approximations to the likelihood function of Lo [32] and Aït-Sahalia[2] and the spectral GMM estimator using the empirical characteristic function presented in Chacko and Viceira[10] and Singleton[37].
} 
is often referred to as the volatility smile. Since option prices are strictly increasing functions of the volatility, it is possible to invert the function globally so as to associate to an option price the volatility for which the Black-Scholes formula would provide accurate pricing.

Implied volatilities can also be defined for more complex pricing models. These models will usually have extra parameters, so that the inversion of the pricing formula in the volatility parameter can be done if one sets values for the other parameters. The value of the implied volatility will therefore be dependent not only on the option price, but also on the values of the other parameters.

However, analytical option pricing formulas are generally difficult to invert and one has to use numerical procedures which are computationally intensive and whose precision has to be controlled. Moreover, implementing integral solutions such as Heston's formula can be very delicate due to divergences of the integrand in regions of the parameter space.

An avenue to avoid both problems is to rewrite option pricing formulas as power series around values of the parameters for which the model can be analytically solved (i.e it has an explicit form in terms of elementary and special functions; not an integral one). This avenue is followed by Lewis[30].

\subsection{Series expansions of option pricing formulas}

Since option prices are continuous in the volatility of volatility parameter $\gamma$, one can expand the pricing formula around a fixed $\gamma$, which we will set to zero, as it corresponds to a deterministic volatility model which we can solve analytically, as we will show. Generally, options will have prices $c\left(S, K, r, T, V_{t}, \beta, \lambda\right)$ at time $t$, where $S$ is the underlying asset's price at this time, $K$ the strike price, $T$ the expiration date, $\beta$ and $\lambda$ are respectively the objective parameters and the volatility risk premium and $V_{t}$ is the volatility at time $t$. The general expansion around $\gamma=0$ of an option pricing formula has the form

$$
c(S, K, r, T, V(t), \beta(\cdot, \gamma), \lambda)=\sum_{j=0}^{\infty} \mu_{j}(S, K, r, T, V(t), \beta(\cdot, 0), \lambda) \gamma^{j},
$$

the notation $\beta(\cdot, \gamma)$ being chosen to exhibit the explicit dependence of the parameter set $\beta, \beta(\cdot, 0)$ meaning that the $\mu_{j}$ 's have no dependence in $\gamma$. If $\gamma \rightarrow 0$, the model has deterministic volatility. For affine stochastic volatility models, it reduces to

$$
\frac{d V_{t}}{d t}=\kappa^{*}\left(\theta^{*}-V_{t}\right)
$$

with the obvious solution

$$
V_{T}=\theta^{*}+\left(V(t)-\theta^{*}\right) e^{-\kappa^{*}(T-t)} .
$$


Hull and White have shown that in non-constant but deterministic volatility models, the option price is given by the Black-Scholes formula evaluated at the mean value of the integrated volatility over the option life;

$$
c(S, K, r, T, V(t), \beta(\cdot, 0), \lambda)=c_{B S}\left(S, K, r, T, \frac{1}{T-t} \overline{\mathcal{V}}_{t, T}\right),
$$

with

$$
\begin{aligned}
\overline{\mathcal{V}}_{t, T} & =\mathrm{E}_{t}\left[\mathcal{V}_{t, T}\right] \\
& =\left[\frac{1}{T-t} \int_{t}^{T}\left[\theta^{*}+\left(V(t)-\theta^{*}\right) e^{-\kappa^{*}(T-s)}\right] d s\right] \\
& =\theta^{*}+\left(V(t)-\theta^{*}\right) \frac{1-e^{-\kappa^{*}(T-t)}}{\kappa^{*}(T-t)} .
\end{aligned}
$$

The first coefficient of the expansion is thus

$$
\mu_{0}\left(S, K, r, T, V_{t}, \beta(\cdot, 0), \lambda\right)=c_{B S}\left(S, K, r, T, \frac{1}{T-t} \overline{\mathcal{V}}_{t, T}\right) .
$$

Other coefficients can be computed in an analogue way (see Appendix A for explicit computations of coefficients of the expansion up to order 6 for the Hulland-White model).

\subsection{Explicit inversion of the pricing formula: Implied volatilities}

One of the main advantages of having at our disposal a series expansion of option pricing formulas, apart from the rapid and simple price computation scheme it provides, is that inverting a series is usually straightforward, so that one can define implied volatilities and compute them very efficiently. As we pointed out earlier, option prices being stricly increasing functions of the volatility, the inversion is always possible. Given the price series expansion (9), assume that given an observed option price $c_{o b s}$, the volatility $V_{t}^{i m p}$ admits the expansion

$$
V_{t}^{i m p}\left(S, K, r, T, c_{o b s}, \beta(\cdot, \gamma), \lambda\right)=\sum_{j=0}^{\infty} \nu_{j}\left(S, K, r, T, c_{o b s}, \beta(\cdot, 0), \lambda\right) \gamma^{j} .
$$

If the observed option price is correctly priced by (9) with volatility being the implied volatility $V_{t}^{i m p}\left(S, K, r, T, c_{o b s}, \beta(\cdot, \gamma), \lambda\right)$, then

$$
\begin{aligned}
c_{o b s} & =c\left(S, K, r, T, V_{t}^{i m p}, \beta(\cdot, \gamma), \lambda\right) \\
& =\sum_{j=0}^{\infty} \mu_{j}\left(S, K, r, T, \sum_{k=0}^{\infty} \nu_{k}\left(S, K, r, T, c_{o b s}, \beta(\cdot, 0), \lambda\right) \gamma^{k}, \beta(\cdot, 0), \lambda\right) \gamma^{j}(18)
\end{aligned}
$$


Having explicit expressions for the $\mu_{j}$ s, in which the volatility parameter will usually enter in a polynomial form, one can solve the above equation order by order in $\gamma$. From these expressions, one can define coefficients $\tilde{\nu}_{j}\left(S, K, r, T, c_{o b s}, \beta(\cdot, 0), \lambda,\left\{\nu_{k}\right\}\right)$ that are such that

$$
c_{o b s}=\sum_{j=0}^{\infty} \tilde{\nu}_{j}\left(S, K, r, T, c_{o b s}, \beta(\cdot, 0), \lambda,\left\{\nu_{k}\right\}\right) \gamma^{j} .
$$

This equation is solved by imposing the conditions

$$
\tilde{\nu}_{0}=c_{o b s} \quad \tilde{\nu}_{j}=0, \forall j \geq 1,
$$

which form a system of polynomial equations for the $\nu_{k}$. This system is easily solved order by order in $k$. Such a procedure can be understood as defining the inverse of a function $f(z)$ as the function $f^{-1}(z)$ which is such that $f\left(f^{-1}(z)\right)=z$. For instance, in mean-reverting stochastic volatility process such as the one we consider here, only the $j=0$ and $k=0$ terms contribute to the zeroth order term in (18), so that the coefficient $\nu_{0}$ can be identified straightforwardly;

$$
c_{o b s}=\tilde{\nu}_{0}=\mu_{0}\left(S, K, r, T, \nu_{0}\left(S, K, r, T, c_{o b s}, \beta(\cdot, 0), \lambda\right), \beta(\cdot, 0), \lambda\right) .
$$

Now, if we define $V_{B S}^{i m p}$ to be the usual Black-Scholes implied volatility, which can always be used instead of the observed option price, we can simplify this equation to

$$
V_{B S}^{i m p}=\theta^{*}+\left(\nu_{0}-\theta^{*}\right) \frac{1-e^{-\kappa^{*}(T-t)}}{\kappa^{*}(T-t)},
$$

so that

$$
\nu_{0}\left(S, K, r, T, V_{B S}^{i m p}, \beta(\cdot, 0), \lambda\right)=\theta^{*}+\left(V_{B S}^{i m p}-\theta^{*}\right) \frac{\kappa^{*}(T-t)}{1-e^{-\kappa^{*}(T-t)}}
$$

In the following, to emphasize that the implied volatility series we defined here is parameter specific, we will refer to it as $V_{t}^{i m p}(\beta, \lambda)$. That is, given an option data set, one can associate an implied volatility time series to every choice of the parameters $\beta, \lambda$. It is only when $\beta=\beta_{0}$ and $\lambda=\lambda_{0}$, the true values of the parameters, that $V_{t}^{i m p}\left(\beta_{0}, \lambda_{0}\right)=V_{t}$. This will be the starting point of our GMM estimation which we will now present.

\subsection{GMM estimation of objective parameters from implied volatilities}

Given daily option prices, the objective parameters $\hat{\beta}$ estimated from high frequency returns and a risk premium $\lambda$, we are now able to construct an implied 
volatility time series. If the risk premium was suitably chosen and the objective parameters the true ones, every option on a given day would have the same implied volatility, so that the implied volatility surface would be flat, with a value equal to the point-in-time volatility $V_{t}$. Since this will not usually be the case, one could use some daily mean value of the observed implied volatilites $V_{t}^{i m p}(\hat{\beta}, \lambda)$ to generate the implied volatility time series. Whether strongly out or in-the-money options should be included in this mean value computation can be debated. We chose to do so when dealing with Monte Carlo generated option prices, but it is not obvious that this would be the reasonable choice when using empirical data.

The implied volatility time series $V_{t}^{i m p}(\hat{\beta}, \lambda)$ can then be considered as a volatility time series, so that one can estimate its parameters $\tilde{\beta}$ using GMM. These estimates will depend on the values $\hat{\beta}$ and $\lambda$, and we shall refer to them as $\tilde{\beta}(\hat{\beta}, \lambda)$. One can then consider the daily volatility series and use the moments of the instantaneous volatility in the moment conditions $f_{t}(\beta)$;

$$
f_{t}(\beta)=\left[\begin{array}{c}
V_{t+1}-\mathrm{E}\left[V_{t+1} \mid \mathcal{F}_{t}\right] \\
V_{t+1}^{2}-\mathrm{E}\left[V_{t+1}^{2} \mid \mathcal{F}_{t}\right] \\
V_{t+1}^{3}-\mathrm{E}\left[V_{t+1}^{3} \mid \mathcal{F}_{t}\right]
\end{array}\right],
$$

where, again, each of these conditional moment conditions are used with two instrumental variables (which are a constant and $V_{t}^{k}$ for the moment condition involving $\left.\mathrm{E}\left[V_{t+1}^{k} \mid \mathcal{F}_{t}\right]\right)$, resulting in six unconditional moment conditions.

One should keep in mind that even though the implied volatility time series is considered as a volatility time series, it will generally provide very poor estimates of the objective parameters if the values of the risk premium and parameters are not suitably chosen. This is hopefully what we are looking for, as this discrepancy between $\hat{\beta}$ and $\tilde{\beta}(\hat{\beta}, \lambda)$ will enable us to estimate the risk premium with precision.

\section{$5 \quad$ Estimating the risk premium}

As we saw in previous sections, we have two different estimations of the objective parameters. The first, from intraday returns, gives a direct way to estimate the parameters via a moment matching procedure and provides $\hat{\beta}$. The second estimation uses option data, the first estimate $\hat{\beta}$, and a value of the risk premium $\lambda$ to provide the estimate $\tilde{\beta}(\hat{\beta}, \lambda)$. As we noted in the end of the last section, a natural way to estimate the risk premium $\lambda$ is to minimize the distance between those two sets of parameters;

$$
\hat{\lambda}=\min _{\lambda}\|\hat{\beta}-\tilde{\beta}(\hat{\beta}, \lambda)\| .
$$


This minimization can be done using standard algorithms. The indirect inference procedure proposed by Gouriéroux, Monfort and Renault [22] deals with similar minimization of distance between two sets of estimate. In their approach, the analogue of $\tilde{\beta}$ is obtained by performing numerical simulations. Even if the approach is different, one could still use the results they give on the optimal choice of metric to estimate $\hat{\lambda}$. Doing so, we obtain two estimates of the objective parameters $\hat{\beta}$ and $\tilde{\beta}(\hat{\beta}, \hat{\lambda})$. Which of the first or second estimate will be more precise over small samples will be discussed in the next section.

\section{A numerical example}

In order to show how the method proposed here can be implemented, we present a numerical study of the Hull-White option pricing model, which can be thought of as the limit of Heston's model in the absence of idiosyncratic leverage effect (that is, $\rho=0$ ). This choice was made mainly because of its simplicity, which will enable the reader to follow the different estimation steps more easily. Heston's model will be considered in a future version of this paper. Eventually, every model which admits a formulation of the option pricing formula in terms of a power series could be considered.

To test the efficiency of our method, we generated, using usual Monte Carlo techniques, several sets of high-frequency price-volatility time series and the associated sets of daily option prices. These prices were computed by using the Hull and White's model specificity that option prices can be expressed as mean values of the Black-Scholes price over the integrated volatility distribution. Explicit expressions for the option prices and implied volatility series up to the sixth order in $\gamma$ can be found in Appendix A.

We chose to study four sets of parameters. The volatility parameters are chosen in the same way as in Bollerslev and Zhou, in order to compare with their results. The four sets of parameters are the following:

- $(\kappa, \theta, \gamma, \lambda)=(0.1,0.25,0.1,0.05)$ : A stationary process with high risk premium.

- $(\kappa, \theta, \gamma, \lambda)=(0.1,0.25,0.1,0.02)$ : The same process with a lower volatility risk premium

- $(\kappa, \theta, \gamma, \lambda)=(0.03,0.25,0.1,0.01)$ : A highly persistent volatility process (nearly unit-root)

- $(\kappa, \theta, \gamma, \lambda)=(0.1,0.25,0.2,0.05)$ : A quasi non-stationary process with high volatility of volatility parameter 
Note that these are daily parameters. As in Bollerslev and Zhou, we normalized $\theta$ so that the yearly volatility is $\frac{\sqrt{240 \times \theta}}{100}$, with 240 being the number of days per year we chose. This means that to $\theta=0.25$ is associated a yearly volatility of $7.74 \%$.

As we just said, each year was defined as 240 days, which were further subdivised in 80 five-minute periods. The quadratic variation of the returns was aggregated over those 80 periods, giving daily integrated volatilies, while option prices where computed from the mid-day instantaneous volatility ${ }^{6}$. Estimation of the implied volatility parameters was performed using GMM for the instantaneous volatility. The moment conditions were $f_{t}(\beta)$, and we used a Newey-West kernel with a lag length of two ${ }^{7}$.

The risk premium structure is chosen as in the Heston's paper, that is, riskneutral (denoted by stars) and objective parameters are related by

$$
\begin{gathered}
\kappa^{*}=\kappa-\lambda \\
\theta^{*} \kappa^{*}=\theta \kappa \\
\gamma^{*}=\gamma .
\end{gathered}
$$

Usually, one would expect $\lambda$ to be positive, so that the asympotic volatility is higher, meaning that option prices will also be. This is however not important in what follows, although all the examples we chose have positive risk premium.

Results of the estimations are provided in the following table. Statistics were obtained by estimating parameters over 250 independent sets of 4-year data. For the estimates $\hat{\beta}$, our results are comparable to those of Bollerslev and Zhou's [9]. The other estimate, $\tilde{\beta}(\hat{\beta}, \hat{\lambda})$ leads to less biased $\kappa$ and $\theta$ estimates, but seems to worsen the $\gamma$ estimation. Since the volatility of volatility coefficient estimation is intimately linked with the data frequency, this is not a surprise. The estimate could be improved if one had access to mid and end-of-day option prices. Finally, the volatility risk premium is also nicely recovered. A more detailed and precise simulation study will be presented in future versions of this paper.

\footnotetext{
${ }^{6}$ Option prices were obtained by simulating volatility trajectories to approximate the integrated volatility distribution. We wanted to avoid using our option pricing formula in order to validate its use. However, if enough trajectories are simulated, the simulated and series expansion price are quasi indistinguishable (at least in the region where the expansion is valid and has enough precision). One could reduce the number of drawn trajectories to add noise to option prices.

${ }^{7}$ We checked that results were quite insensitive to this choice
} 


\begin{tabular}{|l|c|c|c|c|}
\hline True Parameters & $\hat{\beta}$ & $\operatorname{RMSE}(\hat{\beta})$ & $\tilde{\beta}(\hat{\beta}, \hat{\lambda})$ & $\operatorname{RMSE}(\tilde{\beta})$ \\
\hline \hline$\kappa=0.1$ & 0.0939 & 0.0245 & 0.0980 & 0.0175 \\
$\theta=0.25$ & 0.2529 & 0.0180 & 0.2515 & 0.0254 \\
$\gamma=0.1$ & 0.1003 & 0.0105 & 0.0986 & 0.0171 \\
$\lambda=0.05$ & & & 0.0500 & 0.0106 \\
\hline$\kappa=0.1$ & 0.0943 & 0.0215 & 0.1005 & 0.0174 \\
$\theta=0.25$ & 0.2525 & 0.0251 & 0.2521 & 0.0202 \\
$\gamma=0.1$ & 0.1011 & 0.0135 & 0.1010 & 0.0186 \\
$\lambda=0.02$ & & & 0.0217 & 0.0068 \\
\hline$\kappa=0.03$ & 0.0336 & 0.0098 & 0.0288 & 0.0073 \\
$\theta=0.25$ & 0.2425 & 0.0521 & 0.2487 & 0.0489 \\
$\gamma=0.1$ & 0.0947 & 0.0090 & 0.1002 & 0.0117 \\
$\lambda=0.01$ & & & 0.0110 & 0.0060 \\
\hline$\kappa=0.1$ & 0.0993 & 0.0253 & 0.1003 & 0.0267 \\
$\theta=0.25$ & 0.2455 & 0.0330 & 0.2481 & 0.0332 \\
$\gamma=0.2$ & 0.1984 & 0.0185 & 0.1829 & 0.0309 \\
$\lambda=0.05$ & & & 0.0529 & 0.0127 \\
\hline
\end{tabular}

Table 1: Estimation procedure results when the daily implied volatilities are used in the GMM procedure 


\section{Concluding Remarks}

In this paper, we proposed a two-step procedure that permits the joint estimation of objective and risk-neutral parameters for stochastic volatility models. This approach uses fully the information available in the sense that it can adapt to different measure frequency for both the option and returns data it uses. This enables one to use high-frequency intraday return measures and daily option data.

The analytical expressions for the moments of integrated volatility in affine stochastic volatility models that one of the authors of this paper derived recently [31] enable us to obtain explicit expansions of the implied volatility, a crucial point in our procedure. Using these, the method is computationally simple since no simulations or numerical function inversions are involved. Moreover, the first results indicate that the method is comparable in precision to others, even though its building blocks have not been optimized.

Many points are still to be adressed. We first intend to generalize the procedure to all affine stochastic volatility models. This is a tedious computational exercice, but involves no conceptual evolution. Many statistical questions have to be looked at concerning the efficiency of the method and how it could be improved. Finally, we wish to be able to generalize the approach to Levy processes, as they were introduced in a series of recent papers by Barndorff-Nielsen and Shephard $[6,7]$.

\section{A Option prices and implied volatility series expansions}

In this first appendix, we present the explicit series expansions for two wellknown option models: the Hull-White and Heston models. While Hull and White's model could be seen as the zero-leverage limit of Heston's model, its formulation in terms of an integral over the integrated volatility conditional distribution simplifies greatly computations, especially since we have analytical expressions for the integrated volatility conditional moments.

\section{A.1 Hull and White's model}

It is widely known, since the pioneering work of Hull and White, that for the class of stochastic volatility models we consider, if there is no leverage, the price of an o ption is the expectation value, over the integrated volatility riskneutral distribution, of the Black and Scholes price with volatility equal to the 
integrated volatility. Coarsely, an European call of maturity $T-t$ and strike price $K$ on an asset valued $S$ at time $t$ has a price

$$
c_{H W}(S, K, t, T, V(t))=\int d P^{\mathcal{V}(t, T)} c_{B S}\left(S, K, t, T, \frac{1}{T-t} \mathcal{V}(t, T)\right),
$$

where $P^{\mathcal{V}(t, T)}$ is the conditional density of the integrated volatility between $t$ and $T$ knowing the instantaneous volatility at time $t$.

To be more concise, we shall denote the Black-Scholes price on an asset of volatility $V$ by $c_{B S}(V)$. The integration over the distribution of volatilities can be approximated by expanding the integrated volatility around its mean. To do so, one needs analytical expressions for the integrated volatility's central moments, which are given in appendix B, and the sensibilities of the Black and Scholes prices to a change in volatility. These sensibilities can be computed without much effort; computations are shown at the end of this section. Using these, we can extend Hull and White's result and show that $c_{H W}(V(t)$ ) (we omit the extra parameters) can be written as (in order to simplify notations, we will write $\overline{\mathcal{V}} \equiv \frac{1}{T-t} \mathrm{E}_{t} \mathcal{V}_{t, T}$, and $\mathcal{V}$ for $\left.\left.\frac{1}{T-t} \mathcal{V}_{t, T}\right)\right)$

$$
\begin{aligned}
& c_{H W}(V(t))= c_{B S}(\overline{\mathcal{V}})+\left.\frac{1}{2} \frac{\partial^{2} c_{B S}(x)}{\partial x^{2}}\right|_{\overline{\mathcal{V}}} \operatorname{Var}(\mathcal{V})+\left.\frac{1}{6} \frac{\partial^{3} c_{B S}(x)}{\partial x^{3}}\right|_{\overline{\mathcal{V}}} \mathrm{E}_{t}\left[(\mathcal{V}-\overline{\mathcal{V}})^{3}\right]+\cdots \\
&= c_{B S}(\overline{\mathcal{V}})+\frac{1}{2} \frac{S \sqrt{T-t} \Phi^{\prime}\left(d_{1}\right)\left(d_{1} d_{2}-1\right)}{4(\overline{\mathcal{V}})^{3 / 2}} \operatorname{Var}(\mathcal{V})+ \\
&+ \frac{1}{6} \frac{S \sqrt{T-t} \Phi^{\prime}\left(d_{1}\right)\left(\left(d_{1} d_{2}-3\right)\left(d_{1} d_{2}-1\right)-\left(d_{1}^{2}+d_{2}^{2}\right)\right)}{8(\overline{\mathcal{V}})^{5 / 2}} \mathrm{E}_{t}\left[(\mathcal{V}-\overline{\mathcal{V}})^{3}\right] \\
&+ \frac{1}{24} \frac{S \sqrt{T-t} \Phi^{\prime}\left(d_{1}\right)}{16(\overline{\mathcal{V}})^{\frac{7}{2}} \times} \\
&\left(\left(d_{1} d_{2}-5\right)\left(d_{1} d_{2}-3\right)\left(d_{1} d_{2}-1\right)+4 d_{1} d_{2}-\right. \\
&\left.\left(d_{1}^{2}+d_{2}^{2}\right)\left(3 d_{1} d_{2}-9\right)\right) \mathrm{E}_{t}\left[(\mathcal{V}-\overline{\mathcal{V}})^{4}\right]
\end{aligned}
$$

where we recall that

$$
\begin{gathered}
d_{1}=\frac{\log (S / K)+\left(r+\frac{\overline{\mathcal{V}}}{2}\right)(T-t)}{\sqrt{\overline{\mathcal{V}}(T-t)}}, \\
d_{2}=d_{1}-\sqrt{\overline{\mathcal{V}}(T-t)}, \\
c_{B S}(\overline{\mathcal{V}})=S \Phi\left(d_{1}\right)-K e^{-r(T-t)} \Phi\left(d_{2}\right),
\end{gathered}
$$


and $\Phi(\cdot)$ denotes the cumulative function of a standard normal distribution. This gives us a closed form series expansion for option prices. The explicit expressions for the moments of integrated volatility are given in Appendix B. As can be seen there, all moments can be organized as polynomials in $\gamma$. If one collects powers of $\gamma$, the option price expansion can be reorganized as

$c_{H W}(V(t))=c_{B S}(\overline{\mathcal{V}})+c_{H W}^{(2)}(V(t)) \gamma^{2}+c_{H W}^{(4)}(V(t)) \gamma^{4}+c_{H W}^{(6)}(V(t)) \gamma^{6}+\mathcal{O}\left(\gamma^{8}\right)$,

where

$$
\begin{aligned}
& c_{H W}^{(2)}(V(t))= \frac{1}{2} \frac{S \sqrt{T-t} \Phi^{\prime}\left(d_{1}\right)\left(d_{1} d_{2}-1\right)}{4(\overline{\mathcal{V}})^{3 / 2}}\left(A_{T-t} V(t)+B_{T-t}\right) \\
& c_{H W}^{(4)}(V(t))= \frac{1}{6} \frac{S \sqrt{T-t} \Phi^{\prime}\left(d_{1}\right)\left(\left(d_{1} d_{2}-3\right)\left(d_{1} d_{2}-1\right)-\left(d_{1}^{2}+d_{2}^{2}\right)\right)}{8(\overline{\mathcal{V}})^{5 / 2}}\left(M_{T-t} V(t)+N_{T-t}\right) \\
&+\frac{1}{24} \frac{S \sqrt{T-t} \Phi^{\prime}\left(d_{1}\right)}{16(\overline{\mathcal{V}})^{\frac{7}{2}}}\left(\left(d_{1} d_{2}-5\right)\left(d_{1} d_{2}-3\right)\left(d_{1} d_{2}-1\right)+4 d_{1} d_{2}-\right. \\
&\left.\left(d_{1}^{2}+d_{2}^{2}\right)\left(3 d_{1} d_{2}-9\right)\right) Q_{T-t} V(t)^{2} \\
& c_{H W}^{(6)}(V(t))= \frac{1}{24} \frac{S \sqrt{T-t} \Phi^{\prime}\left(d_{1}\right)}{16(\overline{\mathcal{V}})^{\frac{7}{2}}}\left(\left(d_{1} d_{2}-5\right)\left(d_{1} d_{2}-3\right)\left(d_{1} d_{2}-1\right)+4 d_{1} d_{2}-\right. \\
&\left.\left(d_{1}^{2}+d_{2}^{2}\right)\left(3 d_{1} d_{2}-9\right)\right)\left(R_{T-t} V(t)+S_{T-t}\right)
\end{aligned}
$$

In the notations of the second appendix, $\overline{\mathcal{V}}=a_{T-t} V(t)+b_{T-t}$. Since the coefficients $A_{T-t}, B_{T-t}, M_{T-t}, N_{T-t}, Q_{T-t}, R_{T-t}, S_{T-t}, a_{T-t}$ and $b_{T-t}$ do not depend either on $\gamma$ or $V(t)$, it will be simple to perform the series inversion. Note however that $d_{1}$ and $d_{2}$ do depend on $V(t)$.

We want to identify the coefficients $\nu_{j}$ of the implied volatility up to order $\gamma^{6}$. Recall that the expansion is defined as

$$
V_{t}^{i m p}\left(S, K, r, T, c_{o b s}, \beta, \lambda\right)=\sum_{j=0}^{\infty} \nu_{j}\left(S, K, r, T, c_{o b s}, \beta(\cdot, 0), \lambda\right) \gamma^{j}
$$

As we pointed out before, if one inserts the expansion (29) in (25) and further expands in powers of $\gamma$, the coefficients $\nu_{j}$ can be identified by restricting the equation to be satisfied order by order in $\gamma$. The zeroth order condition is thus

$$
c_{o b s}=c_{B S}\left(V_{B S}^{i m p}\right)=c_{B S}\left(a_{T-t} \nu_{0}+b_{T-t}\right) .
$$

This means that, as we announced earlier,

$$
\nu_{0}=\frac{V_{B S}^{i m p}-b_{T-t}}{a_{T-t}} .
$$


The expressions being heavy, we will only show how one can extract the second order in gamma contribution. This can be done by truncating the series (29) to

$$
V_{t}^{i m p}\left(S, K, r, T, c_{o b s}, \beta, \lambda\right)=\frac{V_{B S}^{i m p}-b_{T-t}}{a_{T-t}}+\nu_{1} \gamma+\nu_{2} \gamma^{2}+\mathcal{O}\left(\gamma^{3}\right)
$$

If one expands the coefficient $c_{H W}^{(2)}\left(V_{t}^{i m p}\right) \gamma^{2}$ in powers of $\gamma$ and retains terms up to the second order, he obtains

$$
\begin{aligned}
& c_{H W}^{(2)}\left(V_{t}^{i m p}\right) \gamma^{2}=\frac{\exp \left(\frac{\left(\frac{V_{B S}^{i m p}(T-t)}{2}+2 \log \left[\frac{S}{K}\right]\right)^{2}}{8 V_{B S}^{i m p}(T-t)}\right)}{32 a_{T-t} \sqrt{2 \pi(T-t)}\left(V_{B S}^{i m p}\right)^{5 / 2}} \times \\
&\left(S\left(-a_{T-t} B_{T-t}+A_{T-t}\left(b_{T-t}-V_{B S}^{i m p}\right)\right) \times\right. \\
&\left.\left(V_{B S}^{i m p}(T-t)\left(4+V_{B S}^{i m p}(T-t)\right)-4 \log \left[\frac{S}{K}\right]^{2}\right)\right) \gamma^{2} \\
&+\mathcal{O}\left(\gamma^{3}\right) .
\end{aligned}
$$

Expanding in the same way $c_{B S}(\overline{\mathcal{V}})$ gives

$$
c_{B S}(\overline{\mathcal{V}})=c_{B S}\left(V_{B S}^{i m p}\right)+\frac{a_{T-t} e^{-\frac{V_{B S}^{i m p}(T-t)}{8}-\frac{\log \left[\frac{S}{K}\right]^{2}}{2 V_{B S}^{i m p}(T-t)}} K T \sqrt{\frac{S}{K}}}{2 \sqrt{2 \pi V_{B S}^{i m p}(T-t)}} \nu_{2} \gamma^{2}+\mathcal{O}\left(\gamma^{3}\right) .
$$

The condition

$$
c_{o b s}=c_{B S}\left(V_{B S}^{i m p}\right)=c_{B S}(\overline{\mathcal{V}})+c_{H W}^{(2)}\left(V_{t}^{i m p}\right) \gamma^{2}
$$

will then be satisfied only if $\nu_{1}=0$ and

$\nu_{2}=\frac{\left(a_{T-t} B_{T-t}+A_{T-t}\left(V_{B S}^{i m p}-b_{T-t}\right)\right)\left(V_{B S}^{i m p}(T-t)\left(4+V_{B S}^{i m p}(T-t)\right)-4 \log \left[\frac{S}{K}\right]^{2}\right)}{16 a_{T-t}^{2}\left(V_{B S}^{i m p}\right)^{2}(T-t)}$.

This gives us the inversion up to the second order in $\gamma^{4}$, since one can show that $\nu_{j}=0$ for all odd $j$ 's. The inversion could be performed for higher orders, but expressions quickly become heavy so that we will not report results here; when considering explicit examples, the coefficient $\nu_{4}$ was also used. 


\section{A.2 Sensibilities of the Black-Scholes call price to volatility}

In this subsection, we show how one can compute the successive derivatives of the Black-Scholes formula with respect to the volatility. Giving us the terms appearing in the Hull and White formula, it enables us to go one step further and thus to take into account the higher moments of the integrated volatility.

Recall that

$$
c_{B S}(V)=S \Phi\left(d_{1}\right)-K e^{-r t} \Phi\left(d_{2}\right),
$$

where

$$
\begin{gathered}
d_{1}=\frac{\log (S / K)+\left(r+\frac{V}{2}\right) t}{\sqrt{V t}}, \\
d_{2}=d_{1}-\sqrt{V t} .
\end{gathered}
$$

We will first derive some useful identities. Deriving explicitely $d_{1}$ and $d_{2}$ with respect to $V$ we obtain

$$
\begin{aligned}
& \frac{\partial d_{1}}{\partial V}=-\frac{1}{2 V} d_{2} \\
& \frac{\partial d_{2}}{\partial V}=-\frac{1}{2 V} d_{1} .
\end{aligned}
$$

Also, since $\Phi(x)=\frac{1}{\sqrt{2 \pi}} \int_{-\infty}^{x} e^{-\frac{y^{2}}{2}} d y$, we have

$$
\begin{aligned}
& \Phi^{\prime}(x)=\frac{1}{\sqrt{2 \pi}} e^{-\frac{x^{2}}{2}} \\
& \Phi^{\prime \prime}(x)=-x \Phi^{\prime}(x) .
\end{aligned}
$$

The surprisingly simple form of the derivatives will enable us to find simple expressions for the derivatives of $c_{B S}(V, t)$. The first one is readily computable:

$$
\begin{aligned}
\frac{\partial c_{B S}(V)}{\partial V} & =S \Phi^{\prime}\left(d_{1}\right) \frac{\partial d_{1}}{\partial V}-K e^{-r t} \Phi^{\prime}\left(d_{2}\right) \frac{\partial d_{2}}{\partial V} \\
& =-\frac{1}{2 V}\left(S \Phi^{\prime}\left(d_{1}\right) d_{2}-K e^{-r t} \Phi^{\prime}\left(d_{2}\right) d_{1}\right)
\end{aligned}
$$

To simplify this further, let's observe that, using the definition of $d_{2}$ in terms of $d_{1}$, we have

$$
\begin{aligned}
\Phi^{\prime}\left(d_{2}\right) & =\frac{1}{\sqrt{2 \pi}} e^{-\frac{d_{2}^{2}}{2}}=\Phi^{\prime}\left(d_{1}\right) \exp \left(\log \frac{S}{K}+r t\right) \\
& =\frac{S}{K e^{-r t}} \Phi^{\prime}\left(d_{1}\right)
\end{aligned}
$$


So that

$$
\begin{aligned}
\frac{\partial c_{B S}(V)}{\partial V} & =-\frac{1}{2 V} S \Phi^{\prime}\left(d_{1}\right)\left(d_{2}-d_{1}\right) \\
& =\frac{1}{2 V} S \Phi^{\prime}\left(d_{1}\right) \sqrt{V t} \\
& =\frac{S \Phi^{\prime}\left(d_{1}\right) \sqrt{t}}{2 \sqrt{V}} .
\end{aligned}
$$

Getting a simple form for the first derivatives enables us to derive the following ones easily. For the second derivative:

$$
\begin{aligned}
\frac{\partial^{2} c_{B S}(V)}{\partial V^{2}} & =\frac{S \sqrt{t}}{2}\left(\frac{-1}{2 V^{\frac{3}{2}}} \Phi^{\prime}\left(d_{1}\right)+\Phi^{\prime \prime}\left(d_{1}\right) \frac{-1}{2 V^{\frac{3}{2}}} d_{2}\right) \\
& =\frac{S \sqrt{t} \Phi^{\prime}\left(d_{1}\right)}{4 V^{\frac{3}{2}}}\left(d_{1} d_{2}-1\right)
\end{aligned}
$$

which is the expression appearing in Hull and White's formula. The third one is computed similarly;

$$
\frac{\partial^{3} c_{B S}(V)}{\partial V^{3}}=\frac{S \sqrt{t} \Phi^{\prime}\left(d_{1}\right)}{8 V^{\frac{5}{2}}}\left\{\left(\left(d_{1} d_{2}-3\right)\left(d_{1} d_{2}-1\right)-\left(d_{1}^{2}+d_{2}^{2}\right)\right\},\right.
$$

One can also compute the fourth derivative

$$
\begin{gathered}
\frac{\partial^{4} c_{B S}(V)}{\partial V^{4}}=\frac{S \sqrt{t} \Phi^{\prime}\left(d_{1}\right)}{16 V^{\frac{7}{2}}}\left\{\left(d_{1} d_{2}-5\right)\left(d_{1} d_{2}-3\right)\left(d_{1} d_{2}-1\right)+4 d_{1} d_{2}\right. \\
\left.-\left(d_{1}^{2}+d_{2}^{2}\right)\left(3 d_{1} d_{2}-9\right)\right\}
\end{gathered}
$$

This permits us to go steps further in the series development and take into account the third and fourth central moments of the integrated volatility.

\section{B Computation Method and Expressions for Moments of Integrated Volatility}

We are interested in the integrated volatility of the following bivariate stochastic system

$$
d\left[\begin{array}{c}
p_{t} \\
V_{t}
\end{array}\right]=\left[\begin{array}{c}
\mu+A V_{t} \\
\kappa\left(\theta-V_{t}\right)
\end{array}\right] d t+\sqrt{V_{t}}\left[\begin{array}{cc}
1 & 0 \\
\rho \gamma & \sqrt{1-\rho^{2}} \gamma
\end{array}\right]\left[\begin{array}{l}
d W_{1}(t) \\
d W_{2}(t)
\end{array}\right] .
$$


Observing that the volatility process doesn't have any explicit price dependence, one can study it separately. It obeys the SDE

$$
d V_{t}=\kappa\left(\theta-V_{t}\right) d t+\gamma \sqrt{V_{t}}\left(\rho d W_{1}(t)+\sqrt{1-\rho^{2}} d W_{2}(t)\right) .
$$

If $-1 \leq \rho \leq 1$, the diffusive part of this stochastic differential equation $\left(\rho d W_{1}(t)+\right.$ $\sqrt{1-\rho^{2}} d W_{2}(t)$ ) is a standard brownian motion (it has mean zero, variance one and null higher cumulants). This means that even in the presence of leverage, if one restricts his attention to the volatility sector, the process becomes

$$
d V_{t}=\kappa\left(\theta-V_{t}\right) d t+\gamma \sqrt{V_{t}} d W_{t},
$$

which will be our starting point to compute integrated volatility moments. It is known that, under such processes, the conditional expectation of the volatility is

$$
\begin{aligned}
\mathrm{E}\left[V_{u} \mid V(t)\right] & =\theta+e^{-\kappa(u-t)}(V(t)-\theta) \\
& \equiv \alpha_{u-t} V(t)+\beta_{u-t},
\end{aligned}
$$

where we defined $\alpha_{v} \equiv e^{-\kappa v}$ and $\beta_{v} \equiv \theta\left(1-e^{-\kappa v}\right)$ [13].

We can use this result to compute the conditional expectation of the integrated volatility;

$$
\begin{aligned}
\mathrm{E}\left[\mathcal{V}_{t, T} \mid V(t)\right] & =\mathrm{E}\left[\int_{t}^{T} V_{u} d u \mid V(t)\right] \\
& =\int_{t}^{T} \mathrm{E}\left[V_{u} \mid V(t)\right] d u \\
& =\int_{t}^{T}\left(\theta+e^{-\kappa(u-t)}(V(t)-\theta)\right) d u \\
& \equiv a_{T-t} V(t)+b_{T-t},
\end{aligned}
$$

with $a_{T-t} \equiv \int_{t}^{T} \alpha_{u-t} d u$ and $b_{T-t} \equiv \int_{t}^{T} \beta_{u-t} d u$. Higher moments of the volatility process can be computed recursively using the formula presented in appendix.

In order to compute higher moments, let us consider the $V_{t}$-dependent random variable $\mathrm{E}\left[\mathcal{V}_{t, T} \mid V(t)\right]$ :

$$
\mathrm{E}\left[\mathcal{V}_{t, T} \mid V(t)\right]=\int_{t}^{T} \mathrm{E}_{t}\left[V_{u}\right] d u
$$

If one defines $G(u, t)=\mathrm{E}_{t}\left[V_{u}\right]$, it is clear from the law of iterated expectations that $G(u, t)$ is a martingale in $t$. Thus, Itô's lemma implies that

$$
d \mathrm{E}\left[\mathcal{V}_{t, T} \mid V(t)\right]=-V_{t} d t+a_{T-t} \gamma \sqrt{V_{t}} d W_{t}
$$


Taking integer powers and expectations on each side we obtain

$$
\mathrm{E}_{t}\left[\left(\mathcal{V}_{t, T}-\mathrm{E}\left[\mathcal{V}_{t, T}\right]\right)^{n}\right]=\gamma^{n} \mathrm{E}_{t}\left[\left(\int_{t}^{T} a_{T-s} \sqrt{V_{s}} d W_{s}\right)^{n}\right] ;
$$

where by $\mathrm{E}_{t}[\cdot]$, we mean $\mathrm{E}\left[\cdot \mid V_{t}\right]$. This formula gives us a way to construct all central moments. The computation of this integral is however far from trivial. The interested reader will find in [31] details of the computation for the third and fourth central moments. We will content ourselves of giving the explicit form of the three first central moments for Feller-like stochastic volatility processes (both models considered here are in that class). The variance has the form;

$$
\operatorname{Var}_{t}\left(\mathcal{V}_{t, T}\right)=\gamma^{2}\left(A_{T-t} V(t)+B_{T-t}\right),
$$

where

$$
\begin{aligned}
& A_{T-t}=\frac{1}{\kappa^{2}}\left(\frac{1}{\kappa}-2 e^{-\kappa(T-t)}-\frac{1}{\kappa} e^{-2 \kappa(T-t)}\right) \\
& B_{T-t}=\frac{\theta}{2 \kappa^{3}}\left(-5+2 \kappa(T-t)+4 e^{-\kappa(T-t)}(1+\kappa(T-t))+e^{-2 \kappa(T-t)}\right) .
\end{aligned}
$$

The third central moment has the form

$$
\mathrm{E}_{t}\left[\left(\mathcal{V}_{t, T}--\mathrm{E}_{t}\left[\mathcal{V}_{t, T}\right]\right)^{3}\right]=\gamma^{4}\left(M_{T-t} V(t)+N_{T-t}\right),
$$

with

$$
\begin{gathered}
M_{T-t}=\frac{3}{2 \kappa^{5}}\left(2+e^{-\kappa(T-t)}(1-2 \kappa(T-t)(1+\kappa(T-t)))\right. \\
\left.-2 e^{-2 \kappa(T-t)}(1+2 \kappa(T-t))-e^{-3 \kappa(T-t)}\right) \\
N_{T-t}=\frac{\theta}{2 \kappa^{5}}\left((-22+6 \kappa(T-t))+3 e^{-\kappa(T-t)}(5+2 \kappa(T-t)(3+\kappa(T-t)))\right. \\
\left.+6 e^{-2 \kappa(T-t)}(1+\kappa(T-t))+e^{-3 \kappa(T-t)}\right) .
\end{gathered}
$$

Finally, the fourth moment can be shown to be

$$
\mathrm{E}_{t}\left[\left(\mathcal{V}_{t, T}-\mathrm{E}_{t}\left[\mathcal{V}_{t, T}\right]\right)^{4}\right]=\gamma^{4}\left(Q_{T-t} V(t)^{2}+R_{T-t} V(t)+S_{T-t}\right),
$$

with

$$
Q_{T-t}=\frac{12}{e^{2 \kappa(T-t)} \kappa^{6}}(\sinh (\kappa(T-t))-\kappa(T-t))^{2} .
$$




$$
\begin{aligned}
R_{T-t}= & \frac{1}{e^{4 \kappa(T-t)} \kappa^{7}}\left[\gamma ^ { 2 } \left(-3+15 e^{4 \kappa(T-t)}-12 e^{2 \kappa(T-t)}(1+\kappa(T-t))\right.\right. \\
& \times(1+2 \kappa(T-t))-6 e^{\kappa(T-t)}(2+3 \kappa(T-t)) \\
& \left.\left.\quad-2 e^{3 \kappa(T-t)}(-6+\kappa(T-t)(3+2 \kappa(T-t)(3+\kappa(T-t))))\right)\right] \\
S_{T-t}= & \frac{\theta}{4 e^{4 \kappa(T-t)} \kappa^{7}}\left[\gamma ^ { 2 } \left(3+24 e^{\kappa(T-t)}(1+\kappa(T-t))+e^{4 \kappa(T-t)}\right.\right. \\
& \times(-279+60 \kappa(T-t))+12 e^{2 \kappa(T-t)}(7+2 \kappa(T-t)(5+2 \kappa(T-t))) \\
& +8 e^{3 \kappa(T-t)}(21+\kappa(T-t)(27+2 \kappa(T-t)(6+\kappa(T-t)))) \\
& \left.+3 \kappa\left(1+4 e^{\kappa(T-t)}(1+\kappa(T-t))+e^{2 \kappa(T-t)}(-5+2 \kappa(T-t))\right)^{2} \theta\right] .
\end{aligned}
$$

These explicit expressions are the ones we used in GMM estimation and option pricing expansions.

\section{B.1 Filtration coarsening and moment conditions}

We will show here how one can go from moment conditions of the type $\mathrm{E}\left[\mathcal{V}_{t, t+1}^{k} \mid \mathcal{F}_{t}\right]$, whose expressions are explicitely dependent on the unobservable instantaneous volatility $V_{t}$, to the conditions $\mathrm{E}\left[\mathcal{V}_{t+1, t+2}^{k} \mid \mathcal{G}_{t}\right]$, who depend solely on the integrated volatility process. Let us recall that the filtration $\mathcal{G}_{t}$ is the filtration linked to the integrated volatility process, and is thus coarser than the filtration $\mathcal{F}_{t}$, linked to the volatility process.

Starting from $\mathrm{E}\left[\mathcal{V}_{t+1, t+2} \mid \mathcal{F}_{t+1}\right]=a V_{t+1}+b$ and knowing that $\mathrm{E}\left[V_{t+1} \mid V_{t}\right]=$ $\alpha V_{t}+\beta$, we have

$$
\begin{aligned}
\mathrm{E}\left[\mathrm{E}\left[\mathcal{V}_{t+1, t+2} \mid \mathcal{F}_{t+1}\right] \mid \mathcal{F}_{t}\right] & =a \mathrm{E}\left[V_{t+1} \mid \mathcal{F}_{t}\right]+b \\
& =a \alpha V_{t}+a \beta+b
\end{aligned}
$$

This can be rewritten as

$$
\mathrm{E}\left[\mathcal{V}_{t+1, t+2} \mid \mathcal{F}_{t}\right]=\alpha \mathrm{E}\left[\mathcal{V}_{t, t+1} \mid \mathcal{F}_{t}\right]+\beta
$$

so that, by the law of iterated expectations, we have

$$
\mathrm{E}\left[\mathcal{V}_{t+1, t+2} \mid \mathcal{G}_{t}\right]=\alpha \mathrm{E}\left[\mathcal{V}_{t, t+1} \mid \mathcal{G}_{t}\right]+\beta
$$

The last equation being written solely in terms of observable quantities, it can be used in a GMM estimation procedure. The same method can be applied to higher moment conditions. Starting from

$$
\operatorname{Var}\left[\mathcal{V}_{t+1, t+2} \mid \mathcal{F}_{t+1}\right]=A V_{t+1}+B
$$


we can write

$$
\begin{aligned}
\left.\mathrm{E}\left[\mathcal{V}_{t+1, t+2}^{2} \mid \mathcal{F}_{t+1}\right]\right] & \left.=\operatorname{Var}\left[\mathcal{V}_{t+1, t+2} \mid \mathcal{F}_{t+1}\right]+\left(\mathrm{E}\left[\mathcal{V}_{t+1, t+2} \mid \mathcal{F}_{t+1}\right]\right]\right)^{2} \\
& =a^{2} V_{t+1}^{2}+(2 a b+A) V_{t+1}+\left(b^{2}+B\right)
\end{aligned}
$$

Taking expectations, we have

$$
\left.\mathrm{E}\left[\mathrm{E}\left[\mathcal{V}_{t+1, t+2}^{2} \mid \mathcal{F}_{t+1}\right]\right] \mid \mathcal{V}_{t}\right]=a^{2} \mathrm{E}\left[V_{t+1}^{2} \mid \mathcal{F}_{t}\right]+(2 a b+A) \mathrm{E}\left[V_{t+1} \mid \mathcal{F}_{t}\right]+\left(b^{2}+B\right)
$$

Replacing the moments of the instantaneous volatility by their explicit expressions and using the formulas we have for the first two moments of integrated volatility, we can rewrite this expression as

$$
\begin{aligned}
\left.\mathrm{E}\left[\mathcal{V}_{t+1, t+2}^{2} \mid \mathcal{F}_{t}\right]\right]=\alpha^{2} \mathrm{E} & {\left[\mathcal{V}_{t, t+1} \mid \mathcal{F}_{t}\right] } \\
+ & \frac{1}{a}\left(a^{2}(C+2 \alpha \beta)+\left(\alpha-\alpha^{2}\right)(2 a b+A)\right) \mathrm{E}\left[\mathcal{V}_{t, t+1} \mid \mathcal{F}_{t}\right] \\
& -\frac{b}{a}\left(a^{2}(C+2 \alpha \beta)+\left(\alpha-\alpha^{2}\right)(2 a b+A)\right) \\
+ & \left(a^{2}\left(D+\beta^{2}\right)+\beta(2 a b+A)+\left(1-\alpha^{2}\right)\left(b^{2}+B\right)\right),
\end{aligned}
$$

where

$$
C=\frac{\gamma^{2}}{\kappa}\left(e^{-\kappa}-e^{-2 \kappa}\right)
$$

and

$$
D=\frac{\gamma^{2} \theta}{2 \kappa}
$$

Coarsening the filtration leads to the desired moment conditions. We shall stop computations here, but it clear that following moment conditions can be computed in the same way.

\section{Heston's Model}

Although it is not widely known, the price of an European call option in Heston's model can also be written as an expectation value of the Black-Scholes option pricing under the stochastic volatility distribution. In fact, Romano and Touzi (1997), have shown that, under the stochastic volatility process

$$
d\left[\begin{array}{l}
S_{t} \\
V_{t}
\end{array}\right]=\left[\begin{array}{c}
\mu_{t} S_{t} \\
\kappa\left(\theta-V_{t}\right)
\end{array}\right] d t+\sqrt{V_{t}}\left[\begin{array}{cc}
S_{t} & 0 \\
\gamma \rho & \gamma \sqrt{1-\rho^{2}}
\end{array}\right]\left[\begin{array}{c}
d W_{t}^{1} \\
d W_{t}^{2}
\end{array}\right],
$$

the call option price $c\left(S_{0}, V_{0}, T\right)$ can be written as

$$
c\left(S_{0}, V_{0}, T\right)=\mathrm{E}\left[c_{B S}\left(S_{0} \quad T\left(V_{0}\right), \bar{V}_{T}\left(V_{0}\right)\right],\right.
$$


where

$$
\begin{gathered}
{ }_{T}\left(V_{0}\right)=\exp \left(\rho \int_{0}^{T} \sqrt{V}_{T} d W_{t}^{1}-\frac{1}{2} \rho^{2} \int_{0}^{T} V_{t} d t\right) \\
\bar{V}_{T} \equiv \frac{1}{T}\left(1-\rho^{2}\right) \int_{0}^{T} V_{t} d t .
\end{gathered}
$$

Although it is attractive, this formula was usually regarded as one who could only be useful by performing simulations. Using Monte Carlo, it provides a quite rapid and precise method to compute option prices. In fact, one can show how the inverse Fourier transform method and this formulation of Heston's model are linked. Performing a numerical inversion of the transform is indeed nothing more than integrating over the distribution of some suitably constructed variable, which can be shown to be linked to integrated volatility and our Girsanov-like initial price.

It is straightforward to show (using Girsanov theorem and simple stochastic calculus) that

$$
\mathrm{E}\left[T\left(V_{0}\right)\right]=1
$$

and

$$
\mathrm{E}\left[\bar{V}_{T}\left(V_{0}\right)\right]=\left(1-\rho^{2}\right)\left(\theta+\left(V_{0}-\theta\right) \frac{1-e^{-\kappa T}}{\kappa T}\right) \equiv \bar{V}_{T}^{*} .
$$

The latter is nothing but $\left(1-\rho^{2}\right)$ times the mean integrated volatility. The identification of these mean values enables us to expand the option pricing formula around these:

$$
c\left(S_{0}, V_{0}, T\right)=\left.\sum_{p, q=0}^{\infty} \frac{1}{p ! q !} \frac{\partial^{p+q} c_{B S}}{\partial V^{p}}\right|_{S_{0}, \bar{V}_{T}^{*}} S_{0}^{q} \mathrm{E}\left[(\quad T-1)^{q}\left(\bar{V}_{T}-\bar{V}_{T}^{*}\right)^{p}\right]
$$

Defining

$$
R^{(p, q)}(S, V, T) \equiv \frac{\left(\frac{\partial}{\partial V}\right)^{p}\left(S \frac{\partial}{\partial S}\right)^{q} c_{B S}}{\frac{\partial c_{B S}}{\partial V}}
$$

this can be rewritten as

$$
c\left(S_{0}, V_{0}, T\right)=\left.\sum_{p, q=0}^{\infty} \frac{R^{(p, q)}\left(S_{0}, \bar{V}_{T}^{*}, T\right)}{p ! q !} \mathrm{E}\left[(T-1)^{q}\left(\bar{V}_{T}-\bar{V}_{T}^{*}\right)^{p}\right] \partial c_{B S} \partial V\right|_{S_{0}, \bar{V}_{T}^{*}} .
$$

In terms of the moneyness

$$
X \equiv \log \left[\frac{S}{K e^{-r T}}\right]
$$


and the mean integrated volatility

$$
Z \equiv \frac{1}{\left(1-\rho^{2}\right)} \bar{V}_{T}^{*}
$$

the first values of $R^{(p, q)}$ are the following:

$$
\begin{aligned}
& R^{(1,0)}=1 \\
& R^{(2,0)}=T\left(\frac{X^{2}}{2 Z^{2}}-\frac{1}{2 Z}-\frac{1}{8}\right) \\
& R^{(1,1)}=\left(-\frac{X}{Z}+\frac{1}{2}\right) \\
& R^{(1,2)}=\left(\frac{X^{2}}{Z^{2}}-\frac{X}{Z}-\frac{1}{4 Z}(4-Z)\right) \\
& R^{(2,2)}=T\left(\frac{1}{2} \frac{X^{4}}{Z^{4}}-\frac{1}{2} \frac{X^{3}}{Z^{3}}-3 \frac{X^{2}}{Z^{3}}+\frac{1}{8} \frac{X}{Z^{2}}(12+Z)+\frac{1}{32} \frac{1}{Z^{2}}\left(48-Z^{2}\right)\right) .
\end{aligned}
$$

We will now show how is able to compute the expectation values $\Gamma^{(p, q)} \equiv$ $\mathrm{E}\left[\left(T_{T}-1\right)^{q}\left(\bar{V}_{T}-\bar{V}_{T}^{*}\right)^{p}\right]$. Obviously, we have $\Gamma^{(0,0)}=1$ and $\Gamma^{(0,1)}=\Gamma^{(1,0)}=0$, since the expansion is around the mean values of $\quad T$ and $\bar{V}_{T}$. Also, the terms of type $\Gamma^{(p, 0)}$ are nothing but the central moments of integrated volatility, which we already know how to compute. We thus will devote our attention to the other terms.

Let us first compute $\Gamma^{(1,1)}$.

\section{DETAILED COMPUTATIONS WILL BE INSERTED HERE.......}

$$
\begin{aligned}
& J_{1}(\kappa, \theta, \rho, V, T)= \begin{cases}\frac{1}{2} \rho V T^{2} & \text { if } \kappa=0 \\
\frac{\rho}{\kappa^{2}}\left((\theta+(1+\kappa T)(\theta-V(0))) e^{-\kappa T}+(\kappa T-2) \theta+V(0)\right) & \text { if not }\end{cases} \\
& J_{2}(\kappa, \theta, \rho, V, T)=0 \\
& J_{3}(\kappa, \theta, \rho, V, T)= \begin{cases}\frac{1}{6} V T^{3} & \text { if } \kappa=0 \\
\frac{\left(\theta-2 V+e^{2 \kappa T}((-5+2 \kappa T) \theta+2 V)+4 e^{\kappa T}(\theta+\kappa T \theta-\kappa T V)\right.}{4 e^{2 \kappa T} \kappa^{3}} & \text { if not }\end{cases} \\
& J_{4}(\kappa, \theta, \rho, V, T)=\left\{\begin{array}{ll}
\frac{1}{6} \rho^{2} V T^{3} & \text { if } \kappa=0 \\
\frac{\rho^{2}\left(\left(6+2 e^{\kappa T}(-3+\kappa T)+\kappa T(4+\kappa T)\right) \theta+\left(-2+2 e^{\kappa T}-\kappa T(2+\kappa T)\right) V\right)}{2 e^{\kappa T} \kappa^{3}} & \text { if not }
\end{array},\right.
\end{aligned}
$$


Putting all this together, we have the following two formulas, which respectively give second-order expansion for the option price and its associated BlackScholes implied volatility.

\section{Option pricing expansion}

$$
\begin{aligned}
c(S, V, T)= & c_{B S}(S, \overline{\mathcal{V}}, T)+\left.\frac{\gamma}{\tau} J_{1}(\kappa, \theta, \rho, V, T) R^{(1,1)} \frac{\partial c_{B S}}{\partial V}\right|_{V=\overline{\mathcal{V}}} \\
& +\gamma^{2}\left(\frac{1}{\tau^{2}} J_{3}(\kappa, \theta, \rho, V, T) R^{(2,0)}\right. \\
& +\frac{1}{\tau} J_{4}(\kappa, \theta, \rho, V, T) R^{(1,2)} \\
& \left.+\frac{1}{2 \tau^{2}}\left(J_{1}(\kappa, \theta, \rho, V, T)\right)^{2} R^{(2,2)}\right)\left.\frac{\partial c_{B S}}{\partial V}\right|_{V=\overline{\mathcal{V}}}+\mathcal{O}\left(\gamma^{3}\right)
\end{aligned}
$$

Below, we show how one can invert the series

$$
\begin{aligned}
V_{B S}^{i m p}(S, V, T)= & v(V, T)+\frac{\gamma}{\tau} J_{1}(\kappa, \theta, \rho, V, T) R^{(1,1)} \\
& +\gamma^{2}\left(\frac{1}{\tau^{2}} J_{3}(\kappa, \theta, \rho, V, T) R^{(2,0)}\right. \\
& +\frac{1}{\tau} J_{4}(\kappa, \theta, \rho, V, T) R^{(1,2)} \\
& \left.+\frac{1}{2 \tau^{2}}\left(J_{1}(\kappa, \theta, \rho, V, T)\right)^{2}\left[R^{(2,2)}-\left(R^{(1,1)}\right)^{2} R^{(2,0)}\right]\right)+\mathcal{O}\left(\gamma^{3}\right)
\end{aligned}
$$

in order to be able to extract an Heston-implied volatility from the observable Black-Scholes implied volatility. Namely, we will identify the coefficient $\lambda_{i}$ such that

$$
V=\lambda_{0}\left(S, V_{B S}^{i m p}, T\right)+\lambda_{1}\left(S, V_{B S}^{i m p}, T\right) \gamma+\lambda_{2}\left(S, V_{B S}^{i m p}, T\right) \gamma^{2}+\mathcal{O}\left(\gamma^{3}\right)
$$

Such a development makes sense, because if $\gamma$ goes to zero, the Heston model becomes a deterministic volatility model (in fact, if $\kappa$ goes to zero, we recover the Black-Scholes model). Computing the coefficient is a straightforward but tedious exercice. We will show how one can compute $\lambda_{0}$ and $\lambda_{1}$.

The procedure is simple; one inserts the series expansion for $V$ in the series expansion for $V_{B S}^{i m p}$, and equates the powers of $\gamma$ (there are powers of $\gamma$ appearing in the denominators; these must be expanded in order to obtain a series expansion in powers of $\gamma$ ). Doing so for the $\gamma$-independent part, we have 


$$
V_{B S}^{i m p}=\frac{\left(1-e^{-\kappa T}\right)\left(\lambda_{0}-\theta\right)}{\kappa T}+\theta
$$

which gives

$$
\lambda_{0}=\theta+\frac{\kappa T\left(V_{B S}^{i m p}-\theta\right)}{1-e^{-\kappa T}} .
$$

One then goes on and identifies the $\gamma^{1}$ terms. Since there is none on the left side, the coefficient of $\gamma$ on the right side must sum up to zero. Doing so, one has

$$
\begin{aligned}
\lambda_{1}= & \frac{\rho}{2\left(1-e^{-\kappa T}\right)^{2} \kappa T V_{B S}^{i m p}} \\
& \times\left(V_{B S}^{i m p} T-2 \log \frac{S}{K}\right) \\
& \times\left(\left(1+e^{2 \kappa T}-e^{\kappa T}\left(2+\kappa^{2} T^{2}\right)\right) \theta-e^{\kappa T} \kappa T\left(e^{\kappa T}-1-\kappa T\right) V_{B S}^{i m p}\right)
\end{aligned}
$$

Higher order terms can be computed in the same way. 


\section{References}

[1] Aït-Sahalia, Y.(1996), Nonparametric Pricing of Interest Rate Derivatives, Econometrica, vol. 64, 527-600.

[2] Aït-Sahalia, Y. (1998) Maximum Likelihood Estimation of Discretely Sampled Diffusions: A Closed-Form Approach, Working Paper, Dpt. of Economics, Princeton University.

[3] Andersen, T. and Bollerslev, T (1998) Answering the Spektics: Yes, Standard Volatility Do Provide Accurate Forecasts, International Economic Review, vol. 53, 219-265.

[4] Andersen, T., Bollerslev, T.,Diebold, F.X. and Ebens, H. (2000) The Distribution of Stock Return Volatility, Working Paper, Dpt. of Economics, Duke University.

[5] Andersen, T., Bollerslev, T.,Diebold, F.X. and Labys, P. (1999) The Distribution of Exchange Rate Volatility, NBER Working Paper, No. 6961.

[6] Barndorff-Nielsen, O.E and Shephard, N. (1998) Aggregation and model construction for volatility models, Working Paper, Nuffield College, Oxford.

[7] Barndorff-Nielsen, O.E and Shephard, N. (1998) Modelling by Lévy processes for Financial Econometrics, Working Paper, Nuffield College, Oxford.

[8] Black, F. and M. Scholes (1973) The pricing of Options and Corporate Liabilities, Journal of Political Economy 81, May, 637-659.

[9] Bollerslev, T. and Zhou, H. (2000) Estimating Stochastic Volatility Diffusion Using Conditional Moments of the Integrated Volatility, Working Paper, Duke University.

[10] Chacko, G. and Viceira, L. (1999) Spectral GMM Estimation of ContinuousTime Processes, Working Paper, Graduate School of Business, Harvard University.

[11] Chernov, M. and Ghysels, E. (2000) A Study Towards a Unified Approach to the Joint Estimation of Objective and Risk Neutral Measures for the Purpose of Options Valuation, Journal of Financial Economics, vol. 56.

[12] Chernov, M., Gallant, R., Ghysels, E. and Tauchen, G. (1999) Estimating Jump-Diffusions with a Panel of Options and Returns, Working Paper, Dpt. of Finance, Pensylvania State University.

[13] Cox, J., Ingersoll Jr., J. and Ross, S. (1985) An intertemporal General Equilibrium Model of Asset Prices, Econometrica, 53, 363-384.

[14] Duffie, D. and Singleton, K. (1993) Simulated Moments Estimation of Markov Models of Asset Prices, Econometrica, vol. 61, 929-52. 
[15] Duffie, D. and Kan, R. (1996) A Yield Factor Model of Interest Rates, Mathematical Finance, 6, 379-406.

[16] Duffie, D., Pan., J. and Singleton, K. (1999) Transform Analysis and Asset Pricing for Affine Jump-Diffusions, Econometrica, forthcoming.

[17] Elerian, O., Siddhartha, C. and Shephard, N. (1998) Likelihood Inference for Discretely Observed Non-Linear Diffusions, Working Paper, Nuffield College, Oxford University.

[18] Engle, R.F. and Lee, G.G.J. (1997) Estimating Diffusion Models of Stochastic Volatility, in "Modeling Stock Market Volatility: Bridging the Gap to Continous Time"(Edited by Rossi P. E), Academic Press, New York

[19] Eraker, B. (1998) MCMC Analysis of Diffusion Models with Application to Finance, Graduate School of Business, University of Chicago.

[20] Gallant, R.A. and Long, J.R. (1997) Estimating Stochastic Differential Equations Efficiently by Minimum Chi-Square, Biometrika, vol. 84, 125-141.

[21] Gallant, R.A. and Tauchen, G.E. (1996) Which Moments to Match?, Econometric Theory, vol.12, 657-681.

[22] Gouriéroux, C., Monfort, A. and Renault, E. (1993) Indirect Inference, Journal of Applied Econometrics, vol. 8, s85-s118.

[23] Gouriéroux, C., Monfort, A. and Renault, E. (1996) Two-Stage Generalized Method of Moments and Applications to Regression with Hetereoskedasticity of Unknown Form, Journal of Statistical Planning and Inference, vol. 50, p. 37-63.

[24] Hansen, L.P. and Scheinkman, J.A (1995) Back to the Future: Generalized Moment Implication for Continuous Time Markov Process, Econometrica, vol.63, $767-804$.

[25] Harrison, M. and Kreps, D. (1979) Martingales and Arbitrage in Multiperiod Securities Markets, Journal of Economic Theory, vol. 20, 381-408.

[26] Heston, S.L. (1993) A Closed-Form Solution for Options with Stochastic Volatility with Applications to Bond and Currency Options, The Review of Financial Studies, 6, No. 2, 327-343.

[27] Hull, J. and White, A. (1987) The Pricing of Options on Assets with stochastic volatility, The Journal of Finance, June, 281-300.

[28] Jacquier, E., Polson, N.G. and Rossi, P.E. (1994) Bayesian Analysis of Stochastic Volatility Models, Journal of Business and Economic Statistics, vol.12, 371-389. 
[29] Kim, S., Shephard, N. and Siddhartha, C. (1998) Stochastic Volatility: Likelihood Inference and Comparison with ARCH models, Review of Economic Studies, vol. 65, 361-94.

[30] Lewis, A.L. (2000) Option Valuation under Stochastic Volatility, Finance Press.

[31] Lewis, M.-A. (2000) Analytical expressions for the moments of the integrated volatility in affine stochastic volatility models, Working Paper, CIRANO.

[32] Lo, A.W. (1988) Maximum Likelihood Estimation fo Generalized Itô Process with Discretely Sampled Data, Econometric Theory, vol.4, 231-247.

[33] Pan, J. (1999) Integrated Time-Series Analysis of Spot and Option Prices, Working Paper, MIT Sloan School of Management.

[34] Pastorello, S., Renault, E. and Touzi, N. (2000) Statistical Inference for Random Variance Option Pricing, Journal of Business and Economic Statistics, vol. 18, 358-367.

[35] Renault, E. and Touzi, N. (1996) Option Hedging and Implied Volatilities in a Stochastic Volatility Model, Mathematical Finance, 6, No.3, July, 279-302.

[36] Renault, E. (1997) Econometric Models of Option Pricing Errors, in "Advances in Economics and Econometrics: Theory and Applications, " Vol. 3, Kreps, D. and Wallis, K. eds., Cambridge University Press.

[37] Singleton, K. (1999) Estimation of Affine Asset Pricing Models Using the Empirical Characteristic Function, Working Paper, Graduate School of Business, Stanford University.

[38] Stanton, R. (1997) A Nonparametric Model of Term Structure Dynamics and the Market Price of Interest Rate Risk, Journal of Finance, vol. 52, 1973-2002. 